\title{
Review
}

\section{Prevention of cancer. A colossal achievement, with much more to do}

The World Cancer Research Fund (WCRF)/American Institute for Cancer Research (AICR) have delivered two very impressive landmark reports: the 'determinants' report on Food, Nutrition, Physical Activity and the Prevention of Cancer in $2007^{(1)}$, which provides the evidence platform on the size and nature of the preventable aspects of various cancers; and the recently published 'solutions' report on Policy and Action for Cancer Prevention. Food, Nutrition and Physical Activity: A Global Perspective ${ }^{(2)}$, which provides more detailed recommendations for action on cancer prevention.

\section{New approaches to evidence}

Both have been enormous international efforts, with the 'determinants' report taking about 6 years and the 'solutions' report taking a bit over an additional year. The scientific panel, the contributors and the secretariat are to be congratulated for these achievements. While the update of the epidemiological evidence for the 2007 report on determinants was able to use a modification of the evidence assessment used in the original $1997 \mathrm{WCRF} / \mathrm{AICR}$ report $^{(3)}$, the 'Policy and Action' report required the development of new and very different approaches for evaluating the evidence. It is clear that the nature of the evidence needed to define the determinants is often very different to that needed to define the solutions ${ }^{(4,5)}$. The members of the scientific panel who were present at the conception and lasted the full 7-8-year gestation of these reports could undoubtedly report on a real evolution (or revolution?) in the conceptualisation and evaluation of the evidence.

The epidemiology of the preventable determinants of cancer ${ }^{(1)}$ converged to a set of 'straightforward' recommendations about staying lean throughout life, limiting the foods that promote unhealthy weight gain and cancer, eating the foods that protect against weight gain and cancer, and staying physically active; a huge complexity of data leading to simple behavioural solutions. Of course, enacting such recommendations in the face of an increasingly obesogenic global food and technological environment is anything but straightforward and those simple behavioural solutions then needed to diverge into the complexity of societal and individual actions and policies.

We are creatures of our environments and the vast, complex food system that feeds what is now almost 7 billion of us a day, and the complex cities that house most of us, cannot change rapidly to create healthier environments. In addition, many of the changes needed for healthier environments are made far more difficult by the colossal commercial drivers which are operating in the opposite direction. These are the powerful vested interests which avidly feed the human propensity to overconsume food, cars and labour-saving technologies and strenuously oppose efforts which might threaten their profits $^{(6)}$. What sort of evidence is required to achieve the changes needed towards healthier environments, given this complex and contentious context?

\section{Systematic evaluation}

The WCRF/AICR have made excellent progress in their new 'Policy and Action' report by creating a systematic evaluation of the types of evidence needed to support specified recommendations for influencing environments and behaviours. In doing so, they have exposed the enormous implementation evidence gaps towards which cancer and chronic disease prevention research efforts need be directed. The report conservatively estimates that about one-third of twelve common cancers could be prevented by appropriate diet, physical activity, body size and associated factors. Add to this the large, preventable proportions of other chronic diseases, such as CVD and type 2 diabetes, and you have a very strong case for dramatically increasing the funding for implementation research - how do we apply the evidence and theories we already have at our disposal? Unfortunately, this seems to be less attractive for both scientists and science funders than the epidemiological, genetic and molecular research that currently dominates the grants and publications in the area.

The report ${ }^{(2)}$ evaluates the evidence for action in the physical environment, economic, social and personal dimensions and, across all of these, the scientific panel have chosen forty-six promising interventions to evaluate. The evaluation considers the political feasibility and acceptability, potential impact, general acceptability, cost, timeframe and transferability of each action. The report does very well on managing the extremely difficult task of making these assessments for the breadth of countries across the globe with very little hard data. Many of the evaluation statements are, therefore, unavoidably general in nature but they certainly add the 'contours' needed for 
priority setting to this otherwise long list of potential actions. Some of the actions is fairly tightly specified - for example, the restriction or probibition of advertising and of unbealthy processed foods to children - whereas others are more non-specific - for example, the reduction of absolute poverty and of income inequalities in all societies.

\section{Need for evidence on the effect of policy action}

In the logic model which defines the proposed pathway for how these interventions will theoretically affect environments, behaviours, BMI and cancer incidence, the right-hand side of the pathway (for example, the influence of BMI on cancer risk) has a relatively good evidence base from the earlier determinants report ${ }^{(1)}$ and the methodology for assessing the 'preventability of cancer' is largely based on these estimates. Where the evidence ground is thin is in the left-hand side of the logic model. What impact will a specific regulation on marketing to children have on the exposure of children to junk food ads and how much will that reduce energy intake and BMI? Similarly, what impact will a specific change to income tax rates or social security payments have on poverty and income inequalities and how will that affect dietary intake and BMI? It is here on the implementation evidence that future research is urgently needed.

The report concludes with a summary of recommended actions for each of the nine key 'actors' they identify from multi-national bodies to people. Again, some contours emerge in the different roles of the various actors and this is helpful in getting us beyond the generic catch-cry of needing a multi-sectoral approach. For some actors, such as industry and media, the recommendations are appropriately centred on actions which would turn some of their more counterproductive activities into positive solutions. Governments attract the longest list of actions and again this is appropriate because they really carry the leadership and policy-making responsibilities.
The actions of other actors, such as civil society, professionals, people and schools, are very dependent on government leadership to be really effective.

Overall, this solutions report from WCRF/AICR is a qualitative leap ahead on how to systematically construct the evidence for preventive action. If it can stimulate research efforts towards filling the implementation evidence gaps, then perhaps the next report (in a few years, after the organisations recover from this current massive effort) will be able to add much tighter quantitative estimates of the impacts of various prevention policies.

Boyd Swinburn

Professor of Population Health

Director WHO Collaborating Centre for Obesity Prevention Deakin University 221 Burwood Highway

Melbourne, Victoria 3125, Australia Email: boyd.swinburn@deakin.edu.au

\section{References}

1. World Cancer Research Fund/American Institute for Cancer Research (2007) Food, Nutrition, Physical Activity, and the Prevention of Cancer: A Global Perspective. Washington, DC: AICR; available at http://www.dietandcancerreport.org

2. World Cancer Research Fund/American Institute for Cancer Research (2009) Policy and Action for Cancer Prevention. Food, Nutrition, and Physical Activity: A Global Perspective. Washington, DC: AICR; available at http://www.dietandcancerreport.org

3. World Cancer Research Fund/American Institute for Cancer Research (1997) Food, Nutrition, and the Prevention of Cancer: A Global Perspective. Washington, DC: AICR.

4. Robinson TN \& Sirard JR (2005) Preventing childhood obesity: a solution-oriented research paradigm. Am J Prev Med 28, 2 Suppl. 2, 194-201.

5. Swinburn B, Gill T \& Kumanyika S (2005) Obesity prevention: a proposed framework for translating evidence into action. Obes Rev 6, 23-33.

6. Moodie R, Swinburn B, Richardson J \& Somaini B (2006) Childhood obesity - a sign of commercial success but market failure. Int J Pediatr Obes 1, 133-138. 